\title{
Relationship between sufficient leisure-time physical activity and happiness: Age stratification perspective
}

Po-Fu Lee

Chinese Culture University

Frank Jing-Horng Lu

Chinese Culture University

Diane L. Gill

University of North Carolina

Yi-Hsiang Chiu

Chinese Culture University

Yu-Hsuan Kuo

Chinese Culture University

Ching-Yu Tseng

Fu Jen Catholic University

Chien-Chang Ho ( 093703@mail.fju.edu.tw)

Fu Jen Catholic University

\section{Research article}

Keywords: Relationship between sufficient leisure-time, physical activity and happiness

Posted Date: January 24th, 2020

DOI: https://doi.org/10.21203/rs.2.21852/v1

License: (c) (i) This work is licensed under a Creative Commons Attribution 4.0 International License.

Read Full License

Version of Record: A version of this preprint was published at Current Psychology on March 8th, 2022. See the published version at https://doi.org/10.1007/s12144-022-02946-x. 


\section{Title page}

Relationship between sufficient leisure-time physical activity and happiness: Age stratification perspective

Po-Fu Lee ${ }^{1,2}$

Email address: f520184fred@yahoo.com.tw

Frank Jing-Horng $\mathrm{Lu}^{1}$

Email address: frankjlu@gmail.com

Diane L. Gill ${ }^{3}$

Email address: dlgill@uncg.edu

Yi-Hsiang $\mathrm{Chiu}^{1}$

Email address: golden0230@gmail.com

Yu-Hsuan Kuo ${ }^{1}$

Email address: yoko833511@hotmail.com

Ching-Yu Tseng ${ }^{2,4}$

Email address: $\underline{015844 @ \text { mail.fju.edu.tw }}$

Chien-Chang $\mathrm{Ho}^{2,4^{*}}$

Email address: 093703@mail.fju.edu.tw

${ }^{1}$ Graduate Institute of Sport Coaching Science, Chinese Culture University, Taipei, Taiwan

${ }^{2}$ Department of Physical Education, Fu Jen Catholic University, New Taipei, Taiwan

${ }^{3}$ Department of Kinesiology, University of North Carolina, Greensboro, NC, United States

${ }^{4}$ Research and Development Center for Physical Education, Health and Information

Technology, College of Education, Fu Jen Catholic University, New Taipei City, Taiwan

*Corresponding author: Chien-Chang Ho, Ph.D., Department of Physical Education, Fu Jen Catholic University, New Taipei City 242, Taiwan

Tel.: +886-2-29053386; Fax: +886-2-29052380; E-mail address: 093703@,mail.fju.edu.tw 


\begin{abstract}
Background: Leisure-time physical activity (LTPA) has been frequently reported as a method to improve happiness. However, the effects of LTPA sufficiency and age differences are less discussed in the literature. The aim of this study was to examine the relationship between LTPA sufficiency and happiness based on an age stratification perspective.
\end{abstract}

Methods: A total of 16,412 data from the national physical activity survey was used for this study. Multivariate logistic regression analyses were applied to examine the odds ratio of LTPA sufficient population on happiness.

Results: LTPA sufficient young adults (25-29 years old) performed 2.5 to 3 times the odds on the happiness while compare to insufficient group. However, an unexpected result indicated the negative association between sufficient LTPA and happiness in 35-39 years old men. Intrapersonal differences (e.g. achievement goals and motivational level) and the environment (social context) might play a fundamental role in the LPTA-happiness relationship.

Conclusions: The present study argued that the context of LTPA and psychological status should be clearly addressed in terms of its benefit on happiness.

Trial registration: This study was approved by the Institutional Review Board of Chung Shan Medical University Hospital (CRREC-104-015), registered on $18^{\text {th }}$ Oct. 2016. 


\section{Background}

Pursuing a state of happiness is an essential goal of life. It related to one's thoughts and feelings that comprise the subjective evaluations of his/her life experiences $[1,2]$. It is also contributing to one's self-development and social progress. Researches indicated that people who are happier are more likely to be productive and creative at work, more prosocial, healthier, lived longer, and have better relationships $[3,4,5]$. Today, however, mental illness has captured a leading cause of sickness absence and work incapacity in most developed countries [6]. Depression, anxiety, and stress-related illness are common threats to one's health or causes functional disability [7]. Unfavorable mental conditions are not only damaging an individual's health and social function, but also gradually impairing social-economic development, and creating public problems. Fortunately, these psychiatric problems are usually treatable; in some cases, they may be preventable [8].

Leisure-time physical activity (LTPA) is one of the significant factors negatively associated with physiological diseases and mental disorders. A meta-analysis on the relationship between LTPA and mental health found that physical activity reduces depression and anxiety in non-clinical populations [9]. Besides, LTPA has been used as one of the approaches to enhance mental or physical conditions. Also, LTPA can be an effective antidepressant treatment for mild to moderate depression compared to traditional medicine and psychotherapy [10]. In other words, LTPA plays an essential role in improving one's health, life qualities, and satisfaction.

In addition, many studies found that there is a positive association between LTPA and happiness. For example, emerging adults (18-25 years old) who were more physically active tended to be happier than those who were physically inactive [11]. Similarly, physically active 
participants had a higher likelihood to report "very happy" in their daily life [12]. A cohort study [13] analyzed the long-term effects of LTPA on happiness and indicated that people who continue participating in LTPA for 2 and 4 years could reduce the likelihood of unhappiness compare to those who were physically inactive. Recently, Zhang and Chen [14] systematically reviewed 23 studies on the association between physical activity and happiness, found that as little as 10 minutes of physical activity per week or one time of doing physical activity per week can increase one's level of happiness. In general, empirical studies supported that engaged in LTPA can improve happiness and quality of life [13,15,16,17].

However, to the best of our knowledge, the research on the association between LTPA and happiness has seldom examined the variation of LTPA on happiness in terms of its frequency, intensity, time, and type (FITT principle), based on the American Colleges of Sport Medicine (ACSM) guidelines. For example, many studies have partly focused on the frequency $[12,18,19]$, durations $[17,20,21]$, and intensity [22] respectively on the relationship between LTPA and happiness. Few studies have measured LTPA by the specific guidelines, none of them discussed on LTPA sufficiency. Although several studies attempted to explore the association between LTPA and happiness, the association between sufficient LTPA and happiness is still unclear. According to the guidelines of the Sports Administration of Taiwan, it is recommended that participants in LTPA should be at least 3 times for more than 30 minutes of moderate to vigor-intensity (heart rate reached $130 \mathrm{bpm}$ or feeling breath quickly and sweating) per week.

Furthermore, although researches on the association between LTPA and happiness produced many meaningful results, these studies have seldom examined the differences of age on this relationship. Specifically, an adult's mental health development is related to two factors across the lifespan [23]. First, personal goals act as guideposts for wellbeing and mental health. 
Second, self-regulation plays an essential role that processes governing goal pursuit and adjustment. These two factors decide how individuals evaluate how they are doing in life and recognize the fact (such as time and energy are always limited) across their life development, eventually reflex to their life satisfaction. On the other hand, indeed, several studies have found that the relationship between happiness and age is presented by a U-shape pattern $[24,25]$. The descent of desires on infeasible aspirations in middle-ages, also the individual adaption to personal strengths and weaknesses, are the common reasons for this result [26]. Although Hyde and colleagues [27] have reviewed the characteristics of LTPA and happiness across the lifespan, the associations between LTPA and happiness have never discussed from the age perspective in adulthood.

Therefore, the present study aims to examine the relationship between sufficient and insufficient LTPA and happiness based on an age stratification perspective among Taiwanese adults. The research data came from a nationwide survey in 2018 .

\section{Materials and Methods}

\section{Data source}

The data from Taiwanese physical activity participation annual survey in 2018 was applied. The data includes a nationwide survey of physical activity behaviors, self-reported health status, personal characteristics, and self-evaluations (includes happiness). Data collection was accomplished by the Institute for Public Opinion, Shih-Hsin University. A computer-assisted telephone interview (CATI) was applied from August to October 2018. Trained and experienced interviewers have employed in the CATI to ensure the quality of data collection. 
All data collections followed by stratified proportional sampling. The sampling population came from citizens aged over 13 years and stratified by 22 districts of the country. The sample consisted of 25,654 residents in total, with sampling errors of $3 \%$ and confidence (CI) of 95\%, which allowed for achieving a sufficient sample size and statistical power. Age was first filtered through the database to ensure the adulthood participants have included in the analysis, followed by the skewness and kurtosis assessment. Outliers of each relevant variables have excluded. Finally, 16,412 of data were included with age between 25 and 64 years old [27]. Due to the telephone-based survey, participants were fully informed about the objective, procedure, and content of this survey. The oral consent has given before the interview. All relevant information has contained by the de-identified secondary dataset and has released for public research purposes. This study was approved by the Institutional Review Board of Chung Shan Medical University Hospital (CRREC-104-015), registered on $18^{\text {th }}$ Oct. 2016.

\section{Data collection}

Variables such as gender, age, education, occupation, self-reported health status, height, weight, body mass index (BMI), and obese status of participants have employed for the demographic assessment. The cut-off points of BMI were suggested by the Health Promotion Administration, Ministry of Health, and Welfare of Taiwan. It includes underweight (BMI < $\left.18.5 \mathrm{~kg} / \mathrm{m}^{2}\right)$, normal weight $\left(18.5 \leq \mathrm{BMI}<24 \mathrm{~kg} / \mathrm{m}^{2}\right)$, overweight $\left(24 \leq \mathrm{BMI}<27 \mathrm{~kg} / \mathrm{m}^{2}\right)$ and obese $\left(\mathrm{BMI} \geq 27 \mathrm{~kg} / \mathrm{m}^{2}\right)$.

\section{Measure of LTPA}

In this study, sufficient LTPA has assessed by a series of questions during the CATI. 
First, the participants were asked to answer their current LTPA participation. The question was like "Have you taken part in any LTPA in the past month?" Positive respondents then have asked for their participating frequency and duration, such as "How many times do you participated in LTPA per week?"; "How many minutes do you usually spend in one time?" The intensity assessment of LTPA referred to participants' self-reported breathing rate and sweating. The question was listed as, "When you are doing LTPA, you usually feel...." Then the respondents were asked to answer one of the structured options such as "no changes on my breath and sweat," "I breathe faster but no sweating," "I breathe normally but sweating," "I breathe quickly and sweating." Participants who reported he/she usually breathe quickly and sweating considered achieved moderate-intensity. Thus, based on the LTPA participating frequency, duration, and intensity, respondents were dichotomized into sufficient and insufficient LTPA groups.

\section{Measure of happiness}

A single item question "Taking all things together, how happy would you say you are?" was used to assess the happiness status of participants [28]. Same approaches have previously applied $[15,16,18,29,30,31,32]$ and has been validated by studies [33,34]. The response scale listed from 1 (very unhappy) to 5 (very happy). Furthermore, all respondents are dichotomized into two groups as happy (i.e., very happy, happy, and fair) and unhappy (i.e., unhappy and very unhappy), according to Wang's approach [35].

\section{Statistical analysis}

Data analyses were performed using IBM SPSS software 25. Student's $t$-test and chi- 
square test were used to analyze the differences in participants' demographic characteristics. Besides, two times of multivariate logistic regression analyses were conducted, included general and age stratification populations, to determine the odds ratios (ORs) of sufficient LTPA to happiness. All regression models in our analysis are followed by the confounding factor adjustment to avoid individual influences. The present study calculated the ORs with 95\% CIs for happiness. All data are expressed as means \pm standard deviation (SD) or frequency percentage. The significance level adopted to reject the null hypothesis was $P<0.05$.

\section{Results}

The demographic characteristics of the research population have shown in Table 1. 16,412 of data were employed and dichotomized into groups regarding their happiness status. More than $87 \%$ of participants are classed into the happy group and demonstrated higher female rate (53.51\%), lower obese rate (15.47\%), higher educated (44.33\% in college), lower jobless rate $(4.02 \%)$, and nearly $80 \%$ self-reported in excellent or good health. Except for the pooled-age, the significant differences have found in all of the demographic data between the two groups.

Table 2 presents the cross-analysis between sufficient LTPA and happiness status. In total, $27.7 \%$ of the happy group and $18.6 \%$ of the unhappy group achieved sufficient LTPA. Men contributed higher sufficient LTPA participating rate in both happy and unhappy group. Significant differences have found $(\mathrm{p}<.001)$ in all comparisons. Table 3 revealed the preliminary results of multivariate logistic regression without age stratification. The results indicated that sufficient LTPA groups are around 1.5 to 2 times the odds of the happiness of insufficient LTPA groups.

Table 4 conducted a series of multivariate logistic regressions to perform the age 
stratification. The result indicated that LTPA sufficient men respondents are more likely to be happy than LTPA insufficient group in age 25-29 (OR: 2.90), 45-49 (OR: 1.80), and 50-54 (OR: 2.66). Moreover, women age 25-29 (OR: 2.66), 35-39 (OR: 4.03), 40-44 (OR: 2.45), 45-49 (OR: 3.43), and 60-64 (OR: 2.01) performed more than 2 times the odds on the happiness of LTPA insufficient group. Surprisingly, men in 35 to 39 years old performed the OR bellows $1(0.54 ; \mathrm{p}: .001)$. The results indicated that individuals who engaged sufficient LTPA in this particular age are only $50 \%$ of odds on happiness while comparing to the insufficient group.

\section{Discussion}

The present study aimed to analyze the associations between sufficient LTPA and happiness among Taiwanese adults in terms of age differences. A representative database has used for the multivariate logistic regression analysis from an age stratification perspective. Although our results generally supported the benefit of sufficient LTPA on happiness, statistic evidence in some ages has not observed. This might be correlated with individual differences and social contexts influences. Our findings provided new information and insights to depict the form of the LTPA-happiness relationship through the adulthood in Taiwan.

First, pooled LTPA sufficient populations performed 1.5 to 2 of odds on happiness than the insufficient group, particularly for women. Our result supports previous researches from a critical perspective on LTPA sufficiency $[11,13,14,18,20,22]$. Second, from the age perspective, the influential ORs have found in both genders age 25 to 29 (OR 2.90 for men, 2.66 for women). This indicated that around 2.5 to 3 times the odds of happiness could be found through a sufficient LTPA participation in such ages. Young adults are typical with the likelihood of 
engaging in regular exercise and increasing their life satisfaction and happiness [36].

Third, although some significant associations could not be found, the results performed the fluctuations of odds on happiness for sufficient LTPA across adulthood. For example, men had the highest OR (2.90) in 25-29 years old, followed by a descending trend until age 35-39 years $(\mathrm{OR}=0.54)$, then ascended to the next peak in age 50-54 years $(\mathrm{OR}=2.66)$. The ORs after 54 years old became slack, and no significance was found. From a social-economic view, Taiwanese men commonly play an essential role in family economics. Men in 30 to 40 years old are usually facing problems such as declining job prospects [37], the rise of precarious work [38], work-family conflict [39]. The burdens and the desires for infeasible aspirations sometimes make their life frustrated [26]. Also, these adverse experiences are commonly limiting one's leisure life and creating problems [40].

Moreover, men's odds ratio in age 35-39 years (0.54) indicated that LTPA sufficient individuals have only half odds on happiness compare to insufficient individuals. Recently, the $3 \times 2$ achievement goal model [41] was applied to the LTPA setting [42]. Their results pointed out that the self-avoidance goal negatively predicted happiness and mediated by dispositional self-control. Pre-middle age men could be facing the same issue. For example, a man who is regularly participating in LTPA to avoid fitness decline or to avoid losses body shape might tally with the scenario. Barriers such as prolong working hours, taking care of children, and pre-occupied with business pressure could impact his dispositional self-control then causes a lower level of happiness [43].

On the other hand, women performed a waved trend with 5 significant odds across adulthood. All of them contributed more than 2 times of odds on the happiness while compare with insufficient group. Two declines have observed between ages 30-34 and 50-59, although 
they did not reach the significant. The happiness of middle-aged women is related to their health status, financial distress, social support, psychological factors (e.g., self-esteem, positive thinking, or self-fulfillment) [44]. The variety of these individualized factors can be huge. Those adverse effects, such as health status and quality of life, might mitigate the benefit of LTPA [45]. Also, on average, Taiwanese women facing the parameter of menopausal symptoms after 50 years old [46]. Physical changes and improper coping strategy significantly decrease quality of life, and also reduce their general life satisfaction [47].

Therefore, although many types of research have indicated the benefit of LTPA on happiness, the present study provides different arguments. Indeed, the LTPA-happiness relationship seems clear from a general perspective. Meaningful benefits have also found in our results, particularly for women. However, the relationship in some ages remains unclear. Intrapersonal differences and social contexts may influence the benefits of LTPA on happiness. Also, psychological status such as achievement goals, motivational level, dispositional selfcontrol should be further investigated.

The strength of the present study was using representative data for analysis. However, there are still some limitations should be addressed. First, we used a single-item question to evaluate participants' general perception of happiness. Although it has been supported by many kinds of research, however, it should consider that the component of happiness may be comprised of the different aspects of life, such as psychological, social, physical, and spiritual happiness. Further, although we have controlled the confounding variables during our analyses, we suggest future studies should include the multi-dimensional measurement of happiness. Moreover, because the present study adopted a cross-sectional study design, a cause-and-effect relationship cannot be guaranteed. Future studies may adopt a longitudinal design to 
understand the cause and effect relationship between LTPA and happiness. Alternatively, use a qualitative design to understand sufficient LTPA-happiness relationship.

\section{Conclusions}

Based on the LTPA sufficiency, the present study provided that: First, women are more beneficial populations in LTPA-happiness relationships across adulthood. Second, men could be struggling against their achievement goals and motivational level while engaging sufficient LTPA, thus decrease their happiness level. Last, especially after middle age, the social context (environment), health and psychological status (intrapersonal factors) play fundamental roles in the LTPA-happiness relationship.

\section{Abbreviations}

ACSM, American Colleges of Sport Medicine; BMI: body mass index; CATI: computerassisted telephone interview; CI: confidence interval; LTPA: leisure-time physical activity; OR: odd ratio; SD: standard deviation

\section{Declarations}

\section{Acknowledgements}

The research data is from the National Physical Fitness Examination Survey Database provided by the Sports Cloud: Information and Application Research Center of Sports for All, 
Sport Administration, Ministry of Education. The interpretation and conclusions contained herein do not represent those of Sport Administration, Ministry of Education.

\section{Funding}

This study was supported by a grant from the Ministry of Education (FJU-A0108153) and the Ministry of Science and Technology (MOST 1105-2627-M-030-002, 106-2627-M030-002, 107-2627-M-030-002) in Taiwan.

\section{Availability of data and materials}

The datasets used and/or analyzed during the current study are not publicly available due to confidentiality but are available from the corresponding author on reasonable request.

\section{Authors' contributions}

PFL and CCH participated in the design, conducted the statistical analyses, interpreted the data, and drafted the manuscript. FJHL supervised the study, assisted in data interpretation, and critically reviewed the manuscript. DLG and YHC helped in conducting the study and revising the manuscript. YHK and CYT helped to manage and analyze the data. 
All authors read and approved the final manuscript.

\section{Ethics approval and consent to participate}

This study was approved by the Institutional Review Board of Chung Shan Medical University Hospital (CRREC-104-015), registered on $18^{\text {th }}$ Oct. 2016.

\section{Consent for publication}

Not applicable.

\section{Competing interests}

The authors declare that they have no competing interests.

\section{Author details}

${ }^{1}$ Graduate Institute of Sport Coaching Science, Chinese Culture University, Taipei City,

Taiwan. ${ }^{2}$ Department of Physical Education, Fu Jen Catholic University, New Taipei City,

Taiwan. ${ }^{3}$ Department of Kinesiology, University of North Carolina, Greensboro, NC, United

States. ${ }^{4}$ Research and Development Center for Physical Education, Health and Information 
Technology, College of Education, Fu Jen Catholic University, New Taipei City, Taiwan.

\section{Reference}

1. Diener, E. (2000). Subjective wellbeing: The science of happiness and a proposal for a national index. American Psychologist, 55, 34-43.

2. Veenhoven R. (2010). Greater happiness for a greater number. Journal of Happiness Studies, 11(5), 605-629.

3. Myers, D.G., \& Diener, E. (2018). The scientific pursuit of happiness. Perspectives on Psychological Science, 13(2), 218-225.

4. Jalali, Z., \& Heidari, A. (2016). The relationship between happiness, subjective wellbeing, creativity and job performance of primary school teachers in Ramhormoz city. International Education Studies, 9(6), 45-52.

5. Oswald, A.J., Proto, E., \& Sgroi, D. (2015). Happiness and productivity. Journal of Labor Economics, 33(4), 789-822.

6. Harvey, S.B., Modini, M., Joyce, S., Milligan-Saville, J.S., Tan, L., Mykletun, A., Bryant, R.A., Christensen, H., \& Mitchell, P.B. (2017). Can work make you mentally ill? A systematic meta-review of work-related risk factors for common mental health problems. Occupational and Environmental Medicine, 74(4), 301-310.

7. Knudsen, A.K., Øverland, S., Aakvaag, H.F., Harvey, S.B., Hotopf, M., \& Mykletun, A. (2010). Common mental disorders and disability pension award: Seven year follow-up of the HUSK study. Journal of Psychosomatic Research, 69, 59-67.

8. Mykletun, A., \& Harvey, S.B. (2012). Prevention of mental disorders: A new era for 
workplace mental health. Occupational and Environmental Medicine, 69, 868-869.

9. Rebar, A.L., Stanton, R., Geard, D., Short, C., Duncan, M.Jr., \& Vandelanotte, C. (2015). A meta-meta-analysis of the effect of physical activity on depression and anxiety in nonclinical adult populations. Health Psychology Review, 9(3), 366-378.

10. Knapen, J., Vancampfort, D., Morie nn, Y., \& Marchal, Y. (2015). Exercise therapy imporves both mental and physical health in patients with major depression. Disability and Rehabilitation, 37(16), 1490-1495.

11. Hyde, A.L., Conroy, D.E., Pincus, A.L., \& Ram, N. (2011). Unpacking the feel-good effect of free-time physical activity: Between- and with-person associations with pleasant-activated feeling states. Journal of Sport and Exercise Psychology, 33(6), 884902.

12. Piqueras, J.A., Kuhne, W., Vera-Villarroel, P., van Straten, A., Cuijpers, P. (2011). Happiness and health behaviours in Chilean college students: A cross-sectional survey. BMC public Health, 11, 443.

13. Wang, F., Orpana, H.M., Morrison, H., de Groh, M., Dai, S., \& Luo, W. (2012). Longterm association between leisure-time physical activity and changes in happiness: Analysis of the prospective national population health survey. American Journal of Epidemiology, 176(12), 1095-1100.

14. Zhang, Z., \& Chen, W. (2019). A systematic review of the relationship between physical activity and happiness. Journal of Happiness Study, 20(4), 1305-1322.

15. Baruth, M., Lee, D.C., Sui, X., Church, T.S., Marcus, B.H., Wilcox, S., et al. (2011). Emotional out-look on life predicts increases in physical activity among initially inactive men. Health Education and Behavior, 38(2), 150-158. 
16. Richards, J., Jiang, X., Kelly, P., Chau, J., Bauman, A., \& Ding, D. (2015). Don’t worry, be happy: Cross-sectional associations between physical activity and happiness in 15 European countries. BMC Public Health, 15(53), 1-8.

17. Stubbe, J.H., de Moor, M.H., Boomsma, D.I., \& de Geus, E.J. (2007). The association between exercise participation and wellbeing: A co-twin study. Preventive Medicine, $44(2), 148-152$.

18. Kye, S.Y., Kwon, J.H., \& Park, K. (2016). Happiness and health behaviors in South Korean adolescents: A cross-sectional study. Epidemiology and Health, 38, e2016022.

19. Moljord, I.E., Moksnes, U.K., Eriksen, L., \& Espnes, G.A. (2011). Stress and happiness among adolescents with varying frequency of physical activity. Perceptual and Motor Skills, 113(2), 631-646.

20. Lathia, N., Sandstrom, G.M., Mascolo, C., \& Rentfrow, P.J. (2017). Happier people live more active lives: Using smartphones to link happiness and physical activity. PLoS ONE, $12(1), \mathrm{e} 0160589$.

21. Rasmussen, M., \& Laumann, K. (2014). The role of exercise during adolescence on adult happiness and mood. Leisure Studies, 33(4), 341-356.

22. Nani, S., Matsouka, O., Tsitskari, E., \& Avgerinos, A. (2017). The role of physical activity in life happiness of Greek drug abusers participating in a treatment program. Sport Sciences for Health, 13(1), 25-32.

23. Hoppman, C.A., \& Gerstorf, D. (2016). Adult lifespan development and optimal mental health. Encyclopedia of Mental Health, 1, 20-22.

24. Wunder, C., Wiencierz, A., Schwarze, J., \& Kuchenhoff, H. (2013). Wellbeing over the life span: Semiparametric evidence from British and German longitudinal data. Review of 
Economics and Statistics, 95(1), 154-167.

25. Schwandt, H. (2015). Unmet aspirations as an explanation for the age U-shape in wellbeing. Journal of Economic Behavior and Organization, 122, 75-87.

26. Blanchflower, D.G., \& Oswald, A.J. (2016). Antidepressants and age: A new form of evidence for U-shaped wellbeing through life. Journal of Economic Behavior and Organization, 127, 46-58.

27. Hyde, A.L., Maher, J.P., \& Elavsky, S. (2013). Enhancing our understanding of physical activity and wellbeing with a lifespan perspective. International Journal of Wellbeing, $3(1), 98-115$.

28. Rasciute, S. \& Downward, P. (2010). Health or Happiness? What is the impact of physical activity on the individual? International Review for Social Sciences, 63(2), 256270.

29. Min, J.H., Lee, E.-Y., Spence, J.C., \& Jeon, J.Y. (2017). Physical activity, weight status and psychological wellbeing among a large national sample of South Korean adolescents. Mental Health and Physical Activity, 12, 44-49.

30. Lera-López, F., Ollo-López, A., \& Sánchez-Santos, J. M. (2016). How does physical activity make you feel better? The mediational role of perceived health. Applied Research in Quality of Life, 12(3), 511-531.

31. Maher, C. A., Toohey, M., \& Ferguson, M. (2016). Physical activity predicts quality of life and happi- ness in children and adolescents with cerebral palsy. Disability and Rehabilitation, 38(9), 865-869.

32. Barreto, P. (2014). Direct and indirect relationships between physical activity and happiness levels among older adults: A cross-sectional study. Aging and Mental Health, 
$18(7), 861-868$.

33. Kye, S.Y., \& Park, K. (2014). Health-related determinants of happiness in Korean adults. International Journal of Public Health, 59, 731-738.

34. Goldberg, D.P., Gater, R., Sartorius, N., Ustun, T.B., Piccinelli, M., \& Gureye, O. (1997). The validity of two versions of the GHQ in the WHO study of mental illness in general health care. Psychological Medicine, 27(1), 191-197.

35. Wang, J. H. (2015). Happiness and social exclusion of indigenous peoples in Taiwan-A social sustainability perspective. PLoS One, 10(2), e 0118305.

36. Grant, N., Wardle, J., \& Steptoe, A. (2009). The relationship between life satisfaction and health behavior: A cross-cultural analysis of young adults. International Journal of Behavioral Medicine, 16, 259-268.

37. Danziger, S., \& Ratner, D. (2010). Labor market outcomes and the transition to adulthood. The Future of Children, 20(1), 133-158.

38. Kalleberg, A. (2009). Precarious work, insecure workers: Employment relations in transition. American Sociological Review, 74(1), 1-22.

39. Lambert, E.G., Qureshi, H., Frank, J., Keena, L.D., \& Hogan, N.L. (2017). The relationship of work-family conflict with job stress among Indian police officers: A research note. Police Practice and Research, 18(1), 37-48.

40. Mansour, S., \& Mohanna, D. (2018). Mediating role of job stress between work-family conflict, work-leisure conflict, and employees' perception of service quality in the hotel industry in France. Journal of Human Resources in Hospitality and Tourism, 17(2), 154174.

41. Elliot, A. J., Murayama, K., \& Pekrun, R. (2011). A 3×2 achievement goal model. Journal 
of Educational Psychology, 103(3), 632-648.

42. Briki, W. (2019). Active exercisers with a higher orientation toward task-approach goal might experience higher happiness: the mediating role of dispositional self- control. International Journal of Sport and Exercise Psychology, published online, 14 Feb. 2019, 1-11. Doi:10.1080/1612197X.2019.1570534.

43. Wai, J.P.M., Wen, C.P., Chan, H.T., Chiang, P.H., Tsai, M.K., Tsai, S.P., \& Chang, H.Y. (2008). Assessing physical activity in an Asian country: Low energy expenditure and exercise frequency among adults in Taiwan. Asia Pacific Journal of Clinical Nutrition, 17(2), 297-308.

44. Kim, S. J., \& Hur, M. H. (2019). Understanding of factors influencing happiness of middle-aged women in Korea based on Maslow's hierarchy of needs. Psychiatry Investigation, 16(7), 539-546.

45. Wu, L. H., \& Tsay, R. M. (2017). The search for happiness: Work experiences and quality of life of older Taiwanese men. Social Indicators Research: An International and Interdisciplinary Journal for Quality-of-Life Measurement, 136(3), 1031-1051.

46. Li, P. C., Tsai, I. J., Hsu, C. Y., Wang, J. H., Lin, S. Z., Ding, D. C., \& Sung, F. C. (2018). Risk of hyperlipidemia in women with hysterectomy - A retrospective cohort study in Taiwan. Scientific Reports, 8, 12956.

47. Ngai, F. W. (2019). Relationship between menopausal symptoms, sense of coherence, coping strategies, and quality of life. Menopause, 26(7), 758-764. 


\section{Supplementary Files}

This is a list of supplementary files associated with this preprint. Click to download.

- Tables.pdf 